\title{
Field Emission Microscopy to Study the Catalytic Reactivity of Binary Alloys at the Nanoscale.
}

\author{
Cédric Barroo ${ }^{1,2}$, Luc Jacobs ${ }^{1}$, Natalia Gilis ${ }^{1}$, Sten V. Lambeets ${ }^{1}$, Sylwia Owczarek ${ }^{1,3}$, Yannick
} De Decker ${ }^{2,4}$, and Thierry Visart de Bocarmé ${ }^{1,2}$

1. Chemical Physics of Materials and Catalysis, Université libre de Bruxelles, Brussels, Belgium

2. Interdisciplinary Center for Nonlinear Phenomena and Complex Systems (CENOLI), Université libre de Bruxelles (ULB), Brussels, Belgium

3. Institute of Experimental Physics, University of Wrocław, Wrocław, Poland

4. Non Linear Physical Chemistry Unit, Université libre de Bruxelles, Brussels, Belgium

Catalysis plays a crucial role in modern industrial applications. The aim in every process involving catalysis is to obtain a high and sustainable conversion along with a high selectivity towards the desired product(s). In the case of heterogeneous catalysis, one of the ways to reach this goal is to design tailored nanoparticles that present a specific composition, shape and morphology. Such engineering of catalysts only works if one understands how the reaction proceeds on different morphologies and how the reaction may induce structural changes. Another way to improve the efficiency relies in the control of the catalytic reaction. For this, the study of the dynamics occurring at the surface of the catalyst is used to determine the reaction mechanism with better accuracy, which in turn opens the way to a rationale for assessing the reproducibility, the predictability and the controllability of the reaction. To improve a catalytic process, a fundamental understanding of the catalytic behavior of the active materials is thus required. Surface science studies had, and still have, a great impact on the understanding of catalytic systems. These studies are mainly performed on catalytic reactions occurring at the surface of pure metals. There is, however, an increasing interest in using alloy catalysts in industrial applications, which calls for in situ studies providing a fundamental understanding of the properties of alloy catalysts.

In this context, we use field ion microscopy (FIM) and field emission microscopy (FEM) to determine the structure and composition of alloys in their catalytically active state, and to study the dynamics of reactions, in real time, during the ongoing processes. Experiments are carried out in a field ion/electron emission microscope. Samples are prepared as sharp tips by electrochemically etching a wire made of the ally of interest. In this study, Pt-Rh samples are prepared in a molten salt mixture of $\mathrm{NaCl} / \mathrm{NaNO}_{3}$. $\mathrm{Au}-\mathrm{Ag}$ samples are also studied and require an aqueous solution of KCN 3M. Samples are cleaned by in situ treatments, and then imaged by FIM before physicochemical treatment and by FEM during the ongoing processes.

The first part of this work is dedicated to the hydrogenation of $\mathrm{NO}_{2}$ by Pt-Rh catalyst. Nitrogen oxides are produced in internal combustion engines. The $\mathrm{NO}_{2}$ hydrogenation reaction is of great importance since $\mathrm{NO}$ is first oxidized to $\mathrm{NO}_{2}$ before being reduced by the catalytic converter. Even though the presence of two different metals may induce an imperfect FIM image (as compared to pure metals), it is possible to observe different crystallographic facets with very good accuracy (Figure 1.b). Previous FEM experiments during the ongoing reaction proved that nonlinear dynamics occur on $\mathrm{Pt}$ and $\mathrm{Rh}[1,2]$. The reactivity of the sample is studied by probing the variations of the brightness pattern (see red circle on Figure 1.b). This enables the observation of bursts of reaction (Figure 1.c), as well as non-linear behaviors on the $\mathrm{Pt}-17.4$ at.\% $\mathrm{Rh}$ alloy at $425 \mathrm{~K}$. This $\mathrm{Pt} / \mathrm{Rh}$ ratio corresponds to the applied formulation of catalytic converters in automobile industry. Our results are the first experimental evidence of 
nonlinear dynamics on an alloy catalyst. The reconstruction of the catalyst under pure oxygen environment is also discussed.

The second part of this work addresses alloy catalysts with dual functionality. The idea in such formulations is to combine a small amount of an active metal to facilitate the formation of reactive intermediates, with a less active phase that transforms these intermediates to desirable products with high selectivity. Au-Ag catalysts are used for selective oxidation reactions [3]. The presence of a trace amount of silver in a gold matrix allows for the dissociation of oxygen, which induces the formation of atomic oxygen. The formation of atomic oxygen is an important step in the catalytic process since the ability to supply it is the key for activity and selectivity on gold surfaces. As a model system of surface oxidant, we studied the interaction of $\mathrm{N}_{2} \mathrm{O}$ with Au-8.8 at.\% Ag alloy, as well as the $\mathrm{N}_{2} \mathrm{O}+\mathrm{H}_{2}$ reaction in the 300-320 K temperature range. The reactive behavior can be observed via the appearance of a specific field emission pattern where $\mathrm{O}(\mathrm{ads})$ and $\mathrm{H}(\mathrm{ads})$ react at the interface of $\{210\}$ facets.

Field emission techniques (FIM and FEM) are powerful imaging techniques and by exploiting the nanoscale resolution of these microscopies, it becomes possible to reach a better understanding of catalytic systems down to the molecular level. The experiments on $\mathrm{Pt}-\mathrm{Rh}$ and $\mathrm{Au}-\mathrm{Ag}$ alloys prove that these techniques can be used to study complex systems, and can bring original results on current and future research topics in catalysis. [4]

\section{References:}

[1] C. Barroo, Y. De Decker, T. Visart de Bocarmé and N. Kruse. Phys. Rev. Lett., 117, (2016), 144501.

[2] C. Barroo, et al. Manuscript in preparation.

[3] J. Biener, et al. ACS Catalysis, 2015, (5), 6263.

[4] C.B., L. J. and S. V. L. thank the Fonds de la Recherche Scientifique (F.R.S.-FNRS) for financial support (Postdoctoral fellowship from FNRS, and PhD grants from FRIA, respectively). S. O. thanks Wallonia-Brussels International for the Bilateral Cooperation Agreement.
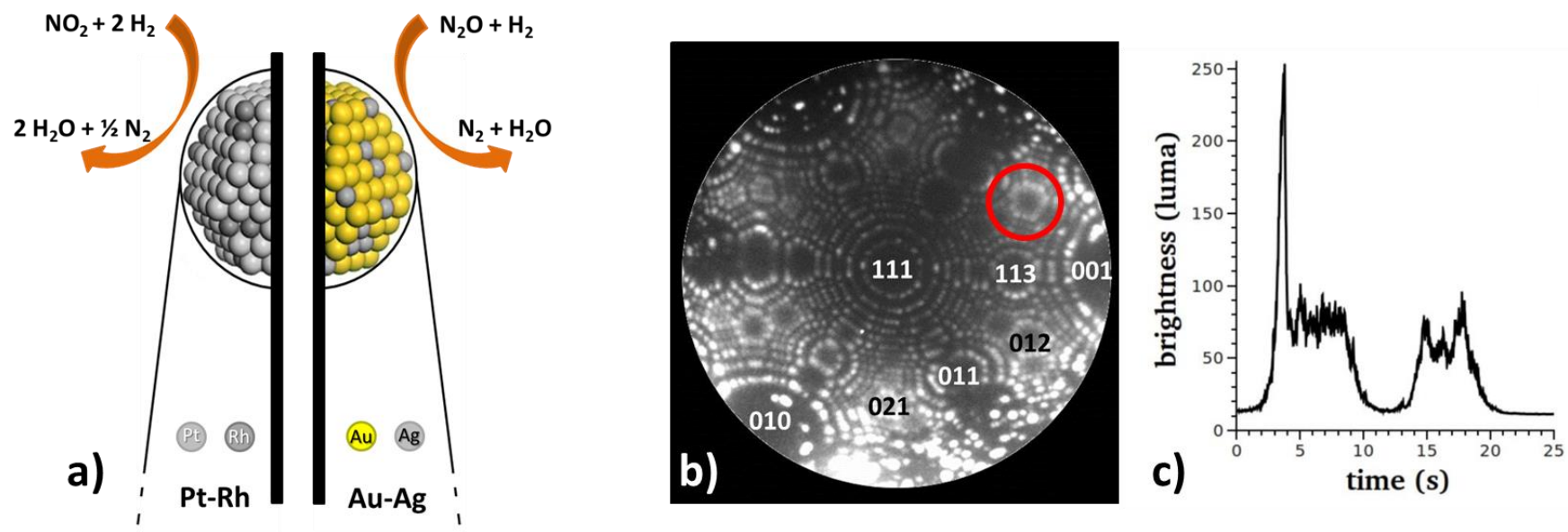

Figure 1. a) Schematic representation of the alloy samples prepared as tips b) Characterization of the Pt-Rh sample at low temperature $(50 \mathrm{~K})$ by field ion microscopy (FIM) and region of analysis corresponding to a (012) facet of approximately $10 \mathrm{~nm}^{2}$. c) Bursts in the brightness signal indicating the presence of reactivity at the surface of the catalyst during the $\mathrm{NO}_{2}$ hydrogenation at $515 \mathrm{~K}$ in FEM mode. 